\title{
Ganoderma lucidum polysaccharide enhances radiosensitivity of hepatocellular carcinoma cell line HepG2 through Akt signaling pathway
}

\author{
YANG YU $^{1}$, LIQI QIAN ${ }^{1}$, NAN DU $^{2}$, YUXIAO LIU ${ }^{3}$, XIAO ZHAO $^{4}$ and XIN ZHANG ${ }^{5}$ \\ Departments of ${ }^{1}$ Traditional Chinese Medicine, ${ }^{2}$ Oncology, ${ }^{3}$ Neurosurgery, ${ }^{4}$ Medical Administration and ${ }^{5}$ Pharmacy, \\ The First Affiliated Hospital of The General Hospital of The People's Liberation Army of China, Beijing 100048, P.R. China
}

Received February 2, 2016; Accepted February 17, 2017

DOI: $10.3892 / \mathrm{etm} .2017 .5340$

\begin{abstract}
Ganoderma lucidum polysaccharide (GLP) is a well-known traditional Chinese medicine, known for its anti-cancer and immunomodulatory properties. The present study aims to investigate whether GLP has a therapeutic effect on hepatocellular carcinoma (HCC) cells exposed to radiation. Immunofluorescence was used to detect the nuclei, the protein expression was measured by western blot analysis and flow cytometry was used to detect the rate of cell apoptosis. GLP treatment was demonstrated to enhance radiation-induced growth inhibition and apoptotic death of HCC cells. At a molecular level, GLP suppressed the activities of DNA repair-associated proteins including ataxia-telangiectasia mutated (ATM) and DNA dependent-protein kinase (DNA-PK) in liver cancer cells under radiation conditions. Furthermore, the addition of an Akt inhibitor elevated the activities of DNA-PK and ATM and attenuated the GLP-induced HepG2 cell injury under the radiation condition. In conclusion, the present study demonstrates that GLP enhances the radiosensitivity of HCC cells via the regulation of Akt signaling pathways, implying a potential therapeutic effect of GLP as a radiation sensitizer in HCC treatment.
\end{abstract}

Correspondence to: Dr Liqi Qian, Department of Traditional Chinese Medicine, The First Affiliated Hospital of The General Hospital of The People's Liberation Army of China, 51 Fucheng Road, Beijing 100048, P.R. China

E-mail: jincuodaoke@sina.com

Dr Nan Du, Department of Oncology, The First Affiliated Hospital of The General Hospital of The People's Liberation Army of China, 51 Fucheng Road, Beijing 100048, P.R. China

E-mail: 36386074@qq.com

Key words: hepatocellular carcinoma, Ganoderma lucidum polysaccharide, radiation, DNA damage

\section{Introduction}

Hepatocellular carcinoma (HCC) is one of the most common causes of cancer-related mortality worldwide (1). It is estimated that of $>1,000,000$ cases of patients diagnosed with $\mathrm{HCC},<5 \%$ survive beyond five years (2). Treatment of HCC primarily consists of liver transplantation, surgical resection and chemotherapy. In addition, ionizing radiation is a well-established and widely used therapeutic modality for patients with HCC (3). However, the majority of the available natural or synthetic compounds are not successful clinically due to their toxicity and side effects. Therefore, novel and effective therapeutics are urgently required for HCC treatment.

The fungi, Ganoderma lucidum (Leyss ex fr) Karst (Lingzhi) has been used in China to treat various human diseases such as hepatitis, bronchitis and tumorigenic diseases (4). Polysaccharides, one of the major categories of the bioactive ingredients of ganoderma lucidum, exhibit multiple biological properties including the prevention of oxidative damage and protection of the liver (5). Previous studies have demonstrated that Ganoderma lucidum polysaccharide (GLP) has antitumor effects, mediated by boosting the activity of host immune cells (6). Furthermore, accumulating evidence supports the hypothesis that GLP exhibits non-toxic and reduced toxicity-related adverse effects compared with chemotherapy or radiotherapy (7). Collectively, these studies suggest that GLP may potentially serve as a chemotherapeutic agent for cancer treatment. In the present study, the effects of GLP on the radiosensitivity of HCC were assessed in addition to its underlying mechanism.

\section{Materials and methods}

Cell culture and treatment. The human HCC cell line HepG2 was obtained from the Chinese Academy of Sciences (Beijing, China). Cells were grown in Dulbecco's Modified Eagle Medium (DMEM; Gibco; Thermo Fisher Scientific, Inc., Waltham, MA, USA) containing $10 \%$ fetal bovine serum (Gibco; Thermo Fisher Scientific, Inc.) supplemented with $100 \mathrm{U} / \mathrm{ml}$ penicillin and $100 \mathrm{mg} / \mathrm{ml}$ streptomycin (Invitrogen; Thermo Fisher Scientific, Inc.). Cells were cultured in in a humidified incubator containing $5 \% \mathrm{CO}_{2}$. For irradiation, 
A
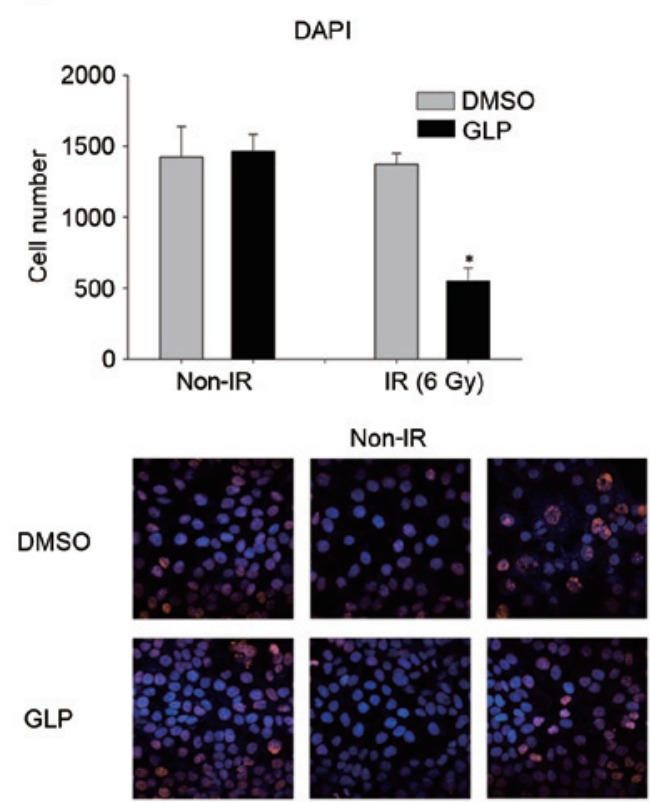

B

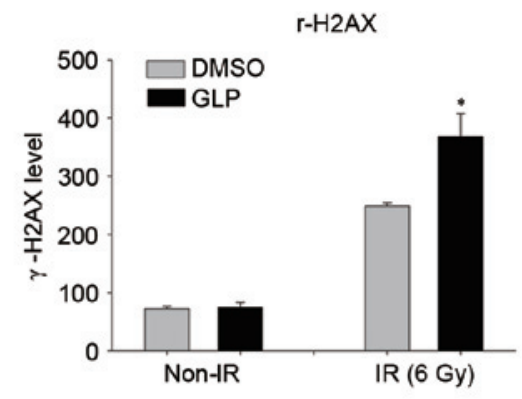

IR

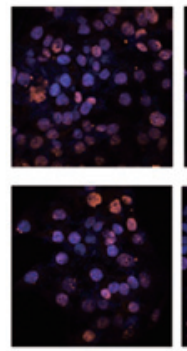

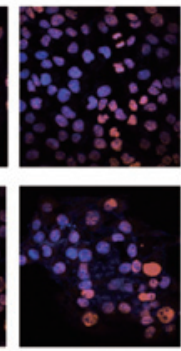

Figure 1. Effect of GLP on radiation-induced inhibition of HepG2 cell growth. HepG2 cells were treated with GLP (10 $\mu \mathrm{M})$ for $72 \mathrm{~h}$ and then with radiation (6 Gy) for $30 \mathrm{~min}$. Treated cells were stained with (A) DAPI (blue) and (B) anti- $\gamma$-H2AX antibody (red). GLP, Ganoderma lucidum polysaccharide; DAPI, 4', 6-diamidino-2-phenylindole; IR, irradiation; DMSO, dimethyl sulfoxide. ${ }^{*} \mathrm{P}<0.05$ vs. DMSO.

HepG2 cells were incubated with GLP (Sigma-Aldrich; Merck $\mathrm{KGaA}$, Darmstadt, Germany) at $37^{\circ} \mathrm{C}$ for $72 \mathrm{~h}$. Then, cells were exposed to radiation using an X-RAD 320 Irradiator (Precision X-Ray, Inc., North Branford, CT, USA) at 6 Gy for $30 \mathrm{~min}$ and collected for the subsequent experiments.

Immunofluorescence. HepG2 cells were fixed with $4 \%$ formaldehyde for $15 \mathrm{~min}$ at room temperature and permeabilized with $0.04 \%$ Triton X-100. Then cells were incubated with $\gamma$-H2AX antibodies (1:1,000; ab2893; Abcam, Cambridge, MA, USA) overnight at $4^{\circ} \mathrm{C}$, washed in PBST for $10 \mathrm{~min}$ three times and incubated with fluorescein isothiocyanate (FITC)-conjugated secondary antibodies $\left(1: 5,000\right.$; ab97050; Abcam) at $37^{\circ} \mathrm{C}$ for $2 \mathrm{~h}$. The nuclei were visualized by staining with 4',6-diamidino-2-phenylindole (DAPI; Sigma-Aldrich; Merck KGaA). Images were captured using fluorescence confocal microscopy (VF1000; Olympus Corporation, Tokyo, Japan).

Apoptosis assay. HepG2 cells were exposed to radiation, GLP, or GLP combined with Akt inhibitor A-674563 at $37^{\circ} \mathrm{C}$ for $72 \mathrm{~h}$ and then collected by centrifugation at 3,000 $\mathrm{x} g$ at room temperature for $15 \mathrm{~min}$. Cell apoptosis was detected by using the Annexin V-FITC apoptosis detection kit (cat. no. 556547; BD Biosciences, Franklin Lakes, NJ, USA) according to the manufacturer's protocol. Apoptosis was determined by calculating the percentage of apoptotic cells relative to the total number of cells.

Western blot analysis. HepG2 cells were incubated with different doses of GLP $(0,10$, and $100 \mu \mathrm{M})$ alone or in combination with Akt inhibitor A-674563 (50 $\mu \mathrm{M})$. Afterwards, HepG2 cells were harvested, washed twice with PBS for $10 \mathrm{~min}$, and lysed in radioimmunoprecipitation assay lysis buffer (Sigma-Aldrich; Merck KGaA). The cell lysates were centrifuged at $12,000 \mathrm{x}$ g for $5 \mathrm{~min}$ at $4^{\circ} \mathrm{C}$. Protein concentration was quantified using a Bradford Protein kit (Bio-Rad Laboratories, Inc., Hercules, CA, USA). Samples with $20 \mu \mathrm{g}$ of protein were separated by $12 \%$ SDS-PAGE, and then the proteins were transferred onto a nitrocellulose membrane. The membrane was blocked with $5 \%$ not-fat milk in tris-buffered saline and Tween-20 (TBST) and incubated with primary antibodies against pDNA-PK (1:200 dilution; sc-101664; Santa Cruz Biotechnology, Inc., Dallas, TX, USA), DNA-PK (1:2,000 dilution; sc-390698; Santa Cruz Biotechnology, Inc.), pATM (1:200 dilution; sc-47739; Santa Cruz Biotechnology, Inc.) and ATM (1:200 dilution; sc-7230; Santa Cruz Biotechnology, Inc.) overnight at $4^{\circ} \mathrm{C}$. The membrane was washed with TBST three times and incubated with a goat anti-mouse IgG secondary antibody that was conjugated with horseradish peroxidase (1:5,000 dilution; sc-5362; Santa Cruz Biotechnology, Inc.) for $1 \mathrm{~h}$. The proteins were detected using an enhanced chemiluminescence detection kit (cat. no. 5384; Roche Diagnostics, Basel, Switzerland). Three replicate blots were analyzed using Quantity One 4.2.1 software (Bio-Rad Laboratories, Hercules, CA, USA).

Statistical analysis. Data were expressed as mean \pm standard deviation and statistical comparisons were performed using one-way analysis of variance. $\mathrm{P}<0.05$ was considered to represent statistically significant differences.

\section{Results}

Effects of GLP on radiation-induced growth inhibition of HepG2 cells. HepG2 cells treated with DMSO or GLP were exposed to radiation at $6 \mathrm{~Gy}$. DAPI staining indicated that administration of GLP had no significant effect on cell number 

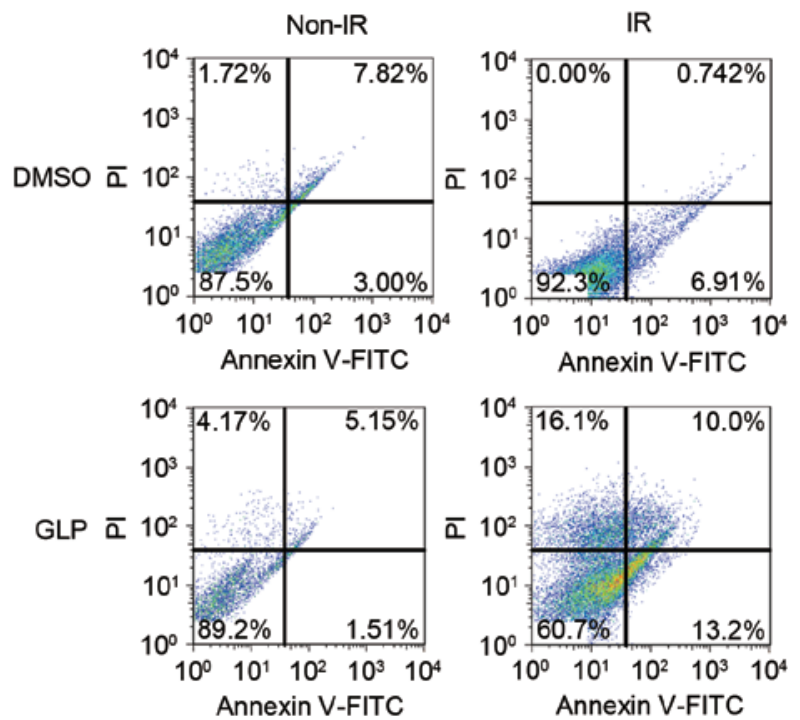

Figure 2. Effects of GLP on radiation-induced apoptosis of HepG2 cells HepG2 cells were treated with GLP $(10 \mu \mathrm{M})$ for $72 \mathrm{~h}$ and then with radiation (6 Gy) for 30 min. Flow cytometry was performed to measure HepG2 cell apoptosis. GLP, Ganoderma lucidum polysaccharide; IR, irradiation; DMSO, dimethyl sulfoxide; FITC, fluorescein isothiocyanate; PI, propidium iodide.

without radiation exposure. However, GLP in combination with radiation significantly reduced the HepG2 cell number compared with the control $(\mathrm{P}<0.05$; Fig. 1A). In addition, immunofluorescence staining for $\gamma-\mathrm{H} 2 \mathrm{AX}$ foci revealed that tumor cells treated with GLP exhibited an increased proportion of nuclei under the radiation condition ( $\mathrm{P}<0.05$; Fig. 1B). These data suggest that GLP treatment enhances radiation-induced growth inhibition of HCC cells.

Effects of GLP on radiation-induced apoptosis of HepG2 cells. It was demonstrated that radiation treatment alone (6 Gy) does not significantly affect the apoptosis rate of HepG2 cells. However, in combination with GLP, radiation treatment significantly promoted HepG2 apoptosis death $(23.2 \%)$ compared with radiation $(7.7 \%)$ or GLP $(6.7 \%)$ alone $(\mathrm{P}<0.05$; Fig. 2). These results demonstrate that GLP co-administration with radiation promotes HCC cell apoptosis under the radiation condition.

Effects of GLP on DNA repair associated proteins expression. In order to investigate the role of GLP in DNA damage upon radiation, the expression of DNA repair associated proteins such as ataxia-telangiectasia mutated (ATM) and DNA dependent-protein kinase (DNA-PK) was assessed. HepG2 cells were incubated with different doses of GLP and then exposed to radiation at $6 \mathrm{~Gy}$. Western blot analysis demonstrated that the phosphorylation of DNA-PK was markedly reduced in GLP-treated HepG2 cells.

In addition, the co-administration of GLP and radiation also inhibited the ATM activities compared with HepG2 cells treated with radiation alone (Fig. 3). These data suggest that GLP may suppress the activity of DNA repair associated proteins in liver cancer cells under the radiation condition.

Akt is associated with the GLP-regulated radiosensitivity in HepG2 cells exposed to radiation. It was then assessed

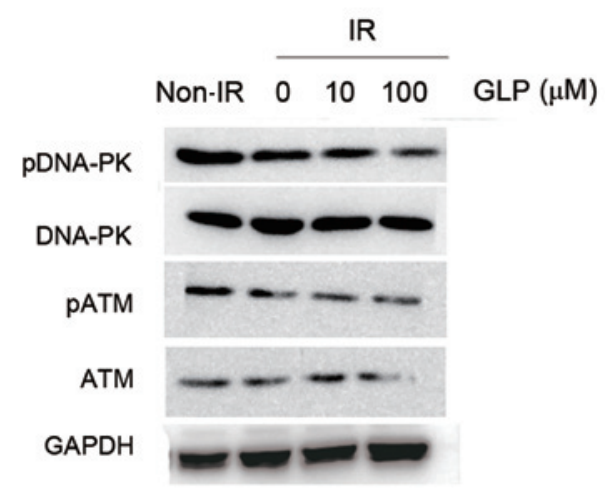

Figure 3. Effects of GLP on DNA repair-associated proteins expression. HepG 2 cells were treated with GLP $(10 \mu \mathrm{M})$ for $72 \mathrm{~h}$ and then with radiation (6 Gy) for $30 \mathrm{~min}$. Western blot analysis was performed to detect the protein expression of ATM and DNA-PK and their phosphorylation levels. GLP, Ganoderma lucidum polysaccharide; ATM, ataxia-telangiectasia mutated; DNA-PK, DNA dependent-protein kinase; IR, irradiation; p, phosphorylated; GAPDH, glyceraldehyde 3-phosphate dehydrogenase.

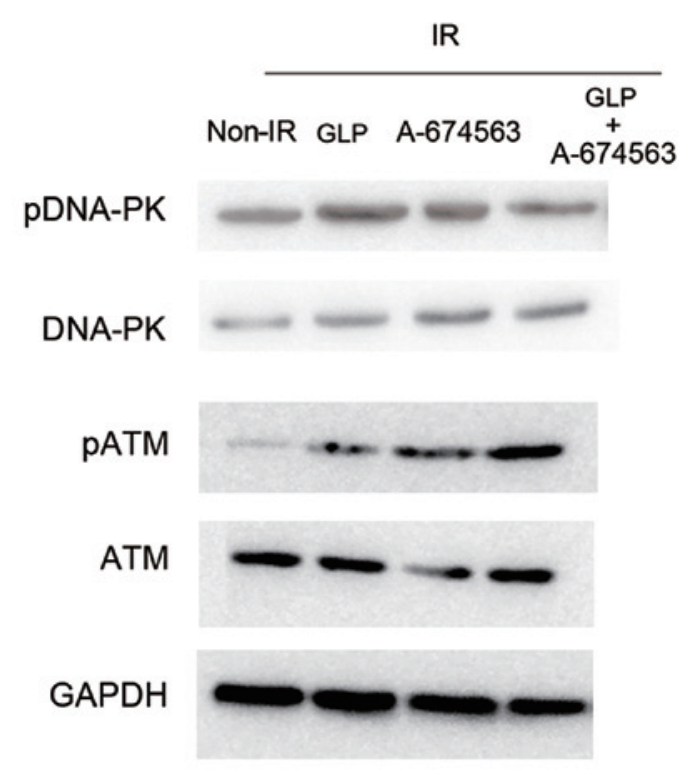

Figure 4. Akt is associated with GLP-regulated ATM activity in HepG2 cells. HepG2 cells were treated with GLP $(10 \mu \mathrm{M})$ alone or combined with Akt inhibitor A-674563 for $72 \mathrm{~h}$ and then with radiation (6 Gy) for $30 \mathrm{~min}$. Western blot analysis was performed to detect the protein expression of ATM and DNA-PK and their phosphorylation levels. Akt, protein kinase B; GLP, Ganoderma lucidum polysaccharide; ATM, ataxia-telangiectasia mutated; DNA-PK, DNA dependent-protein kinase; pDNA-PK, phosphorylated DNA-PK; pATM, phosphorylated-ATM; GAPDH, glyceraldehyde 3-phosphate dehydrogenase.

whether Akt was associated with GLP-mediated ATM activity in HepG2 cells exposed to radiation. HepG2 cells were incubated with Akt inhibitor A-674563 alone or in combination with GLP. Western blot analysis indicated that the phosphorylation level of DNA-PK and ATM was elevated following treatment with A-674563 (Fig. 4). In addition, it was demonstrated that GLP treatment enhanced the apoptotic death of HepG2 cells under radiation compared with DMSO-treated cells (11.13 vs. 8.81\%). However, addition of the Akt inhibitor suppressed the GLP-induced HepG2 cell injury under radiation 

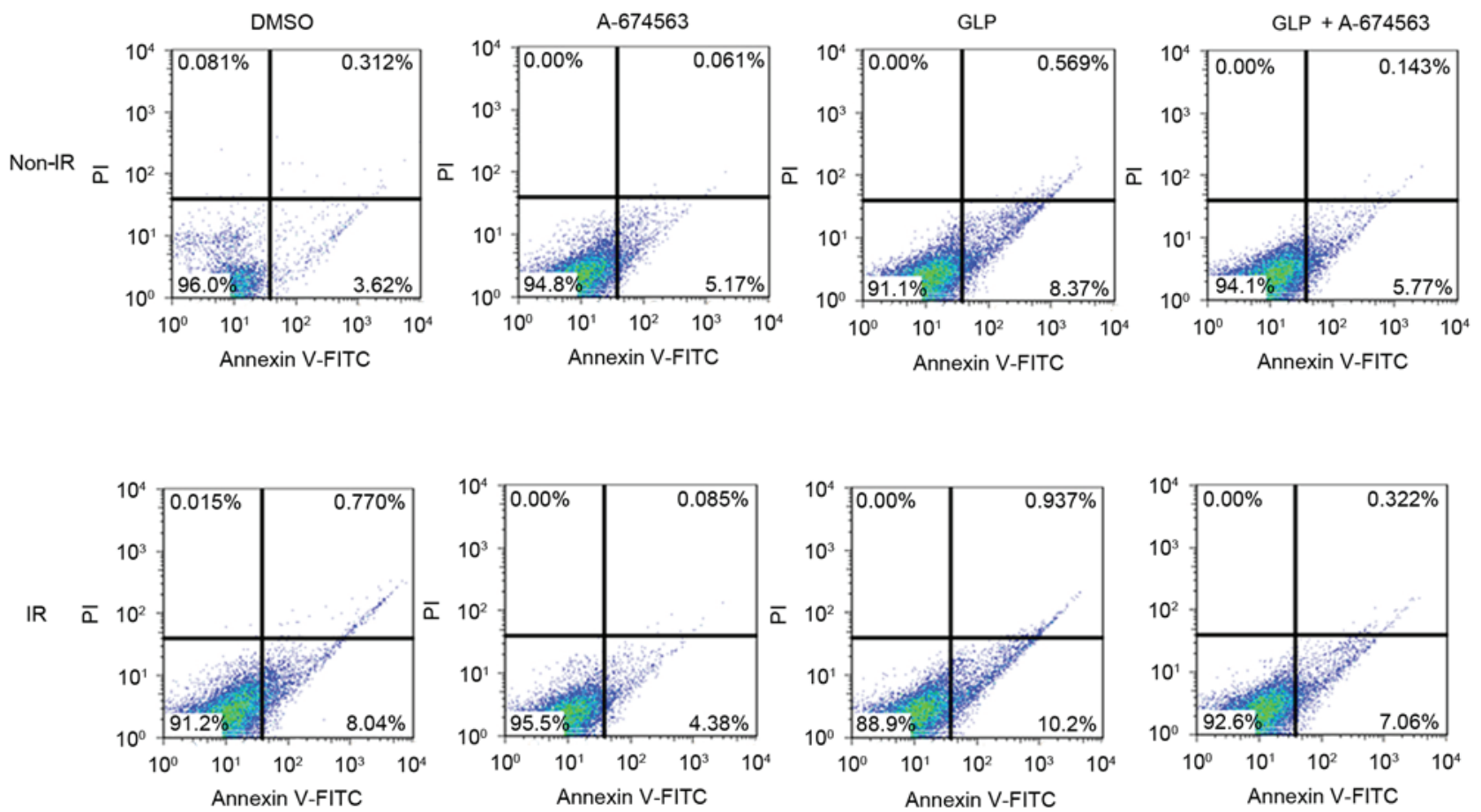

Figure 5. Akt participates in GLP regulated apoptosis in HepG2 cells. HepG2 cells were treated with GLP (10 $\mu$ M) alone or combined with Akt inhibitor A-674563 for $72 \mathrm{~h}$ and then with radiation (6 Gy) for $30 \mathrm{~min}$. Flow cytometry was performed to measure HepG2 cell apoptosis. Akt, protein kinase B; GLP, Ganoderma lucidum polysaccharide; DMSO, dimethyl sulfoxide; IR, irradiation; FITC, fluorescein isothiocyanate; PI, propidium iodide.

condition (7.38 vs. 11.13\%; Fig. 5). These results suggest that Akt participates in GLP-mediated radiosensitivity of HepG2 cells exposed to radiation.

\section{Discussion}

Over the past 30 years, a wide range of traditional Chinese herbs and botanical formulations have been identified as radiosensitizers in cancer treatment (8-10). A number of these even have undergone clinical trials and exhibited favorable therapeutic effects with safety and lower toxicity (8). GLP, an extract of a basidiomycete fungus, possesses various pharmacologic properties including antitumor effects $(5,11,12)$. In the current study, treatment with GLP was demonstrated to significantly promote the apoptosis of HepG2 cells under radiation condition, which may be mediated by the Akt signaling pathway. These findings suggest that GLP has a potential radiosensitization effect on HepG 2 cells exposed to radiation.

Polysaccharides widely exist in microorganisms, algae, plants and animals. Together with polynucleotides and proteins, they are essential biomacromoleules in the biological processes and serve critical roles in cell growth, adhesion, and apoptosis (13). Previously, polysaccharides extracted from natural sources have attracted attention in the field of cancer therapy due to their antitumor properties (14). In the present study, GLP treatment was identified to promote radiation-induced growth inhibition and apoptotic death of HCC cells.

DNA damage response (DDR) serves a critical role in the maintenance of genome integrity (15). During cancer therapy, DDR attenuates the efficiency of radio- or chemotherapeutic agents and thus induces drug resistance of tumor cells to genotoxic stress (16). Therefore, inhibition of DNA repair-associated enzymes may be a promising strategy to increase the sensitivity of ionizing radiation on cancer cells. Cancer cells exposed to radiation exhibit DNA lesions and double strand breaks, which are repaired by ATM and DNA-PK (17). In order to examine the effects of GLP on DDR in HCC cells, the protein expression of ATM and DNA-PK in HepG2 cells exposed to radiation was measured. Results indicated that the phosphorylation of ATM and DNA-PK was markedly suppressed following the administration of GLP, suggesting that GLP may inhibit radiation-induced DNA repair.

The Akt signaling pathway has been demonstrated to regulate multiple biological activities, including cell proliferation, apoptosis and differentiation $(18,19)$. Upregulation of Akt is observed in numerous types of cancer, and it may be associated with uncontrolled cell growth (20). In addition, the Akt signaling pathway has been implicated in a number of processes associated with cell cycle regulation, including DNA replication and damage repair (21). As demonstrated in Figs. 4 and 5, the addition of Akt inhibitor was demonstrated to elevate the activities of DNA-PK and ATM and suppress the GLP-induced HepG2 cell injury under radiation condition. These results indicate that Akt signaling regulated the GLP-mediated radiosensitivity in HepG2 cells exposed to radiation.

In conclusion, the present study demonstrated that GLP treatment may enhance the radiosensitivity of HCC cells via regulation of the Akt signaling pathway. These findings suggest that GLP may be developed into a potential radiation sensitizer to improve the therapeutic efficacy of HCC. 


\section{References}

1. McGlynn KA,Petrick JL and London WT: Global epidemiology of hepatocellular carcinoma: An emphasis on demographic and regional variability. Clin Liver Dis 19: 223-238, 2015.

2. Jemal A, Bray F, Center MM, Ferlay J, Ward E and Forman D: Global cancer statistics. CA Cancer J Clin 61: 69-90, 2011.

3. Lau WY and Lai EC: Hepatocellular carcinoma: Current management and recent advances. Hepatobiliary Pancreat Dis Int 7: 237-257, 2008

4. Xu Z, Chen X, Zhong Z, Chen L and Wang Y: Ganoderma lucidum polysaccharides: Immunomodulation and potential anti-tumor activities. Am J Chin Med 39: 15-27, 2011.

5. Guo L, Xie J, Ruan Y, Zhou L, Zhu H, Yun X, Jiang Y, Lü L, Chen K, Min Z, et al: Characterization and immunostimulatory activity of a polysaccharide from the spores of Ganoderma lucidum. Int Immunopharmacol 9: 1175-1182, 2009.

6. Lin ZB and Zhang HN: Anti-tumor and immunoregulatory activities of Ganoderma lucidum and its possible mechanisms. Acta pharmacologica Sinica 25: 1387-1395, 2004.

7. Zhou X, Lin J, Yin Y, Zhao J, Sun X and Tang K: Ganodermataceae: Natural products and their related pharmacological functions. Am J Chin Med 35: 559-574, 2007.

8. Liu ZL, Zhu WR, Zhou WC, Ying HF, Zheng L, Guo YB, Chen JX and Shen XH: Traditional Chinese medicinal herbs combined with epidermal growth factor receptor tyrosine kinase inhibitor for advanced non-small cell lung cancer: A systematic review and meta-analysis. J Integr Med 12: 346-358, 2014.

9. Jia L, Ma S, Hou X, Wang X, Qased AB, Sun X, Liang N, Li H, Yi H, Kong D, et al: The synergistic effects of traditional Chinese herbs and radiotherapy for cancer treatment. Oncol Lett 5: 1439-1447, 2013.

10. Yang L, Wei DD, Chen Z, Wang JS and Kong LY: Reversal effects of traditional Chinese herbs on multidrug resistance in cancer cells. Nat Prod Res 25: 1885-1889, 2011.

11. Sun Z, Huang K, Fu X, Zhou Z, Cui Y and Li H: A chemically sulfated polysaccharide derived from Ganoderma lucidum induces mitochondrial-mediated apoptosis in human osteosarcoma MG63 cells. Tumour Biol 35: 9919-9926, 2014.
12. Tie L, Yang HQ, An Y, Liu SQ, Han J, Xu Y, Hu M, Li WD, Chen AF, Lin ZB and Li XJ: Ganoderma lucidum polysaccharide accelerates refractory wound healing by inhibition of mitochondrial oxidative stress in type 1 diabetes. Cell Physiol Biochem 29: 583-594, 2012.

13. Pillai TG, Maurya DK, Salvi VP, Janardhanan KK and Nair CK: Fungal beta glucan protects radiation induced DNA damage in human lymphocytes. Ann Transl Med 2: 13, 2014.

14. Zeng G, Shen H, Tang G, Cai X, Bi L, Sun B, Yang Y and Xun W: A polysaccharide from the alkaline extract of Glycyrrhiza inflata induces apoptosis of human oral cancer SCC-25 cells via mitochondrial pathway. Tumour Biol 36: 6781-6788, 2015.

15. Korwek Z, Sewastianik T, Bielak-Zmijewska A, Mosieniak G, Alster O, Moreno-Villanueva M, Burkle A and Sikora E: Inhibition of ATM blocks the etoposide-induced DNA damage response and apoptosis of resting human T cells. DNA Repair (Amst) 11: 864-873, 2012.

16. Nestal de Moraes G, Bella L, Zona S, Burton MJ and Lam EW: Insights into a Critical Role of the FOXO3a-FOXM1 Axis in DNA Damage Response and Genotoxic Drug Resistance. Curr Drug Targets 17: 164-177, 2016.

17. Liu S, Opiyo SO, Manthey K, Glanzer JG, Ashley AK, Amerin C, Troksa K, Shrivastav M, Nickoloff JA and Oakley GG: Distinct roles for DNA-PK, ATM and ATR in RPA phosphorylation and checkpoint activation in response to replication stress. Nucleic Acids Res 40: 10780-10794, 2012.

18. Ourique F, Kviecinski MR, Felipe KB, Correia JF, Farias MS, Castro LS, Grinevicius VM, Valderrama J, Rios D, Benites J, et al: DNA damage and inhibition of akt pathway in mef-7 cells and ehrlich tumor in mice treated with 1,4-naphthoquinones in combination with ascorbate. Oxid Med Cell Longev 2015: 495305, 2015

19. Davis WJ, Lehmann PZ and Li W: Nuclear PI3K signaling in cell growth and tumorigenesis. Front Cell Dev Biol 3: 24, 2015.

20. Liu Z, Zhu G, Getzenberg RH and Veltri RW: The upregulation of PI3K/Akt and MAP kinase pathways is associated with resistance of microtubule-targeting drugs in prostate cancer. J Cell Biochem 116: 1341-1349, 2015.

21. King SM, Quartuccio SM, Vanderhyden BC and Burdette JE: Early transformative changes in normal ovarian surface epithelium induced by oxidative stress require Akt upregulation, DNA damage and epithelial-stromal interaction. Carcinogenesis 34: $1125-1133,2013$. 